\title{
Zur physiologischen Involution des regenerierten Schwanzes von Kaulquappen
}

\author{
Von \\ Tatsuo Fukai \\ (Aus dem Anatomischen Institut der Kaiserlichen Universität zu Kyoto: \\ Prof. Dr. Ch. Ogawa).
}

Bei der Metamorphose tritt Verkürzung des Schwanzes (physiologische Involution) an der Kaulquappe auf. Bezüglich ihres Beginns und ihrer Geschwindigkeit herrscht gewöhnlich eine gewisse Regelmässigkeit. Die Verkürzung fängt nämlich im allgemeinen vor oder nach der Entbindung der Vorderextremitäten an, und zwar geht sie im anfänglichen Stadium ziemlich langsam, im späteren dagegen verhältnismässig schnell vor sich und im ganzen vollzieht sie sich etwa in einer Woche.

Es handelt sich in diesem Falle aber um einen sich normal entwickelnden Schwanz, nicht um eine Anomalie.

Wie verhält es sich nun bei der Verkürzung des Schwanzes unter abnormen Bedingungen z.B. bei Transplantation oder Regeneration? Wird der Involutionsprozess der gleiche sein? Was den transplantierten Schwanz betrifft, so habe ich schon nachgewiesen, dass die Involution mit derselben Regelmässigkeit wie beim normalen stattfindet.

Rawin (1923) berichtete, dass der Regenerationsprozess immer hemmend auf die Involution des Schwanzes einwirkt. Doch hat niemand anscheinend über die Rückbildung des Regenerates selber Mitteilung gemacht.

Nun habe ich Bufolarven, deren Körperlänge ca. 4,5 mm betrug, als Material gewählt, ihren Schwanz an der Basis abgeschnitten und ihn wieder aus dem Schnittstumpf regenerieren lassen. Nach dem Abschneiden wuchs der Schwanz verhältnismässig gut und zwar bis zur Hälfte des Kontroll-Schwanzes. Doch ist der ganze Teil dieses wieder gewach- 
senen Schwanzes nicht als regeneriert zu betrachten, sondern nur das Kaudalstück, das ungefähr $\mathrm{T} / 3$ oder $1 / 2$ desselben ausmacht. Also darf man, streng genommen, nur dieses Kaudalstück aufnehmen, wenn man die Verkürzung des regenerierten Schwanzes beobachten will. Diese tritt nun aber immer in dem ganzen Schwanz gleichzeitig auf. Deshalb ist es sehr schwer, sie nur in dem regenerierten Kaudalstück isoliert zu untersuchen. Es wurde darum in unserem Fall der ganze Schwanz beobachtet.

Auch an diesem regenerierten Schwanz begann die Verkürzung in der Hauptsache vor oder nach der Entbindung der Vorderextremitäten d.h. weder früher noch später als am normalen. Und zwar geht die Verkürzung auch im anfänglichen Stadium verhältnismässig langsam und im späteren ziemlich schnell vor sich. Ungefähr in 6 oder 7 Tagen ist sie vollendet. Die Pigmentierung des Schwanzes tritt hierbei ebenfalls einige Tage vor der Aussprossung der Vorderbeine auf. Das zeigt, dass keine besonderen Unterschiede zwischen dem regenerierten und dem normalen vorhanden sind.

Ferner habe ich die gleiche Untersuchung auch an dem aus dem Transplantat regenerierten Schwanz angestellt. Zu diesem Zweck habe ich Bufolarven, deren Körperlänge ca. 4,5 mm betrug, gewählt, um die Schwanzknospe (ca. I mm) von einem anderen Tier desselben Stadiums an ihre linke Kiemengegend zu transplantieren (Homoioplastische Transplantation), sie am 2. Tag nach der Transplantation an der Basis wieder abgeschnitten und regenerieren lassen.

Bei den best regenerierten Exemplaren betrug der Schwanz $1 / 4$ der Länge des Empfängerschwanzes. In diesem Fall wird das linke Vorderbein etwas vorzeitig entbunden. Diese Tatsache scheint mir von Interesse zu sein, und ich möchte in einer späteren Mitteilung genauer darauf eingehen.

Die Verkürzung beginnt hierbei auch fast vor oder nach der Entbindung der $r$. Vorderextremität und vollendet sich in etwa 5 oder 6 Tagen, indem sie im anfänglichen Stadium verhältnismässig langsam und im späteren ziemlich schnell vor sich geht. Man bemerkt also keine besonderen Unterschiede bei der Verkürzung des aus dem Transplantat regenerierten Schwanzes und der des Empfängerschwanzes.

Mit der Pigmentierung verhält es sich ebenso wie beim normalen Schwanz. Nur gelangt in diesem Fall der Schwanz etwa einige Tage früher zur Bildung des Restes, der auch bald nach dem Leben auf dem Lande vollkommen verloren geht. Das kommt wohl daher, denke ich, dass dieser regenerierte Schwanz vor der Metamorphose sehr viel kürzer als der des Empfängers war. 
Zur physiologischen Involution des regenerierten Schwanzes von Kaulquappen. 385

Die obigen zwei Arten von Experimenten machen es deutlich, dass in bezug auf das Verhalten des Schwanzes bei der physiologischen Involution fast keine bemerkenswerten Unterschiede zwischen dem regenerierten Schwanz und dem normalen sich ergeben. Mit anderen Worten reagieren die Regenerate keineswegs abweichend bezüglich der die physiologische Involution veranlassenden Momente im Vergleich zu dem sich normal entwickelnden Organ.

\section{Schluss.}

Bei der Verkürzung des Schwanzes (physiologische Involution) verhält sich der regenerativ gewachsene Schwanz bezüglich des Beginus und der Geschwindigkeit ebenso wie der normal gewachsene (bei Bufo vulgaris).

Zum Schluss spreche ich meinem hochverehrten Lehrer, Herm Prof. Dr. Ch. Ogawa, meinen herzlichsten Dank für seine mir stets erwiesene Güte aus.

\section{Literatur.}

1) Fukai, T. Über die mit dem Empfängerschwanz synchrone metamorphotische Rückbildung des transplantierten Schwanzes von Bufolarven. Fol. anat. jap. Bd. 12, 1934.

2) Rawin, W. Regeneration und Involution. Anat. Anz. Bd. 57, 1923.

3) Weiss, P. Entwicklungsphysiologie der Tiere. Dresden und I.eipzig 1930. 